RIBOSOMES

Protein Gene Expression

from our Cell Biology Correspondent

IT is hardly surprising that cells require complex machinery in order to synthesize proteins, which are after all large, specific molecules, but whether a decade or so ago many biochemists anticipated the extreme heterogeneity of the structural proteins of the ribosome may be doubted. The fact is, however, that in a $70 \mathrm{~S}$ Escherichia coli ribosome there are some fifty-odd species of structural proteins and such heterogeneity poses several intriguing questions. Why are so many different sorts of proteins required and what do they do? And how are the corresponding structural genes arranged in the $E$. coli genome such that their expression can be coordinated and regulated? That these genes are expressed in a coordinate manner is indisputable, of course, for in bacteria the number of ribosomes per cell is directly proportional to the growth rate.

The obvious first thought is that the structural genes of ribosomal proteins might be organized into operons and the clustering of at least some of these genes is at least consistent with that idea. But the experimental approaches involving the isolation of deletion and nonsense mutations of structural genes and mutations of operators and promoters, which have been used to characterize conventional operons, are clearly inappropriate and unrewarding when it comes to analysing the organization and regulation of ribosomal protein genes because drastic mutational changes in these genes are bound to be lethal. Clearly, the problem demands of bacterial geneticists the sort of ingenuity which Nomura and Engbaek (Proc. US Nat. Acad. Sci., 69, 1526; 1972) encouragingly bring to bear upon it.

Nomura and Engbaek have exploited stable, partial diploid strains of $E$. coli which are heterozygous for the region of the $E$. coli chromosome which includes the spectinomycin, streptomycin, erythromycin and fusidic acid loci at which there can be sensitive or resistant alleles. These loci specify respectively the $\mathrm{S} 5$ and S12 proteins of $30 \mathrm{~S}$ ribosomal subunits, a protein of the $50 \mathrm{~S}$ subunits and the elongation factor $G$. The particular strain they have used carries on the chromosome the markers $\arg G^{-}$, ery , $s p c^{\mathrm{s}}, s t r^{\mathrm{s}}, f u s^{\mathrm{s}}, \operatorname{aro} B^{-}, \operatorname{malT}^{-}$in that order, and an episome carries the markers $\operatorname{ar} G^{+}, e r y^{\mathrm{r}}, \quad s p c^{\mathrm{r}}, \operatorname{str}^{\mathrm{r}}, f u s^{\mathrm{r}}, \operatorname{aro} B^{+}$, mal $T^{+}$. Because the sensitivity alleles are dominant over those conferring resistance the cells have a sensitive phenotype to all four antibiotics.

To determine whether these four loci, which specify ribosomal proteins and an elongation factor, are part of a single transcriptional unit and therefore expressed coordinately, Nomura and Engbaek took advantage of that most useful phage, Mu, which can lysogenize an $E$. coli cell by integrating at random throughout the bacterial chromosome. If phage $\mathrm{Mu}$ integrates in the middle of an operon, the expression of those cistrons distal to the promoter and the $\mathrm{Mu}$ genome is blocked but the cistrons lying between the promoter and the prophage are expressed normally.

Assuming that the four loci in question are part of one transcriptional unit, if $\mathrm{Mu}$ integrates in one of them those receiving or distal to the prophage will be inactivated whereas those proximal to the prophage will be unaffected. And because of the resistance alleles carried in the cell by the episome as soon as one or more dominant chromosomal alleles are inactivated the cell will acquire a resistant phenotype. If each of the four loci is transcribed as a discrete unit, however, each, to be inactivated, must receive a $\mathrm{Mu}$ genome.

Having predicted the various possible outcomes, Nomura and Engbaek infected their partial diploid cells with $\mathrm{Mu}$ phage and analysed the pattern of drug resistance in the infected cultures. All their results are consistent with just one of the predictions which says that the four loci are indeed part of a single transcriptional unit or operon in which the "promoter" is to the left of the four loci $e r y^{\mathrm{s} / \mathrm{r}}, s p c^{\mathrm{s} / \mathrm{r}}, s r^{\mathrm{s} / \mathrm{r}}, f u \mathrm{~s}^{\mathrm{s} / \mathrm{r}}$. The behaviour of spontaneous resistant mutants is also consistent with this model; for example, more than half the spontaneous erythromycin resistant mutants are also resistant to the other three drugs and may well therefore carry polar nonsense mutations. Furthermore, the ribosomes from $\mathrm{Mu}$ induced mutants resistant to all four drugs behave, as expected, in a cell free system making protein.

These data clearly indicate that these four loci form part of some sort of transcriptional unit, and Nomura and Engbaek, who comment that a Belgian group has also found that the $s p c^{\mathrm{s} / \mathrm{r}}$, $s t r^{8 / \mathrm{r}}$ loci are part of transcriptional unit, suggest that many if not all the structural genes of ribosomal proteins are probably in this one massive "operon". Whether the transcription of this ribosomal protein operon is regulated in the way that the transcription of conventional operons is regulated is but one of the fascinating questions which, by further exploitation of partial diploid strains, may soon be answered.

\section{ARTIFICIAL INTELLIGENCE \\ Understanding Program}

from our

Experimental Psychology Correspondent "Understanding Natural Language" is not a bad title for a scientific article, but when the article in question describes a computer program that actually performs this feat, it is even more impressive. The editor of Cognitive Psychology has made a sound decision in devoting a whole issue of the journal (3, 1 ; 1972) to an article by $\mathrm{T}$. Winograd of the Artificial Intelligence Laboratory at Massachusetts Institute of Technology. All the indications are that work in artificial intelligence is becoming extremely important for psychology.

\title{
A Structure for Thermolysin
}

A SET of three articles in next Wednesdaty's Nature New Biology (July 12), from the laboratories of Neurath in Seattle and Matthews at the University of Oregon, report the sequence and $2.3 \AA$-resolution X-ray structure of the proteolytic enzyme, thermolysin. Thermolysin is a bacterial metalloenzyme of molecular weight 35,000 , which contains both zinc and calcium, and is unrelated to the serine and the thiol proteases. The sequence shows some polarization of charged residues. It is not related to that of carboxypeptidase, a protease that it resembles in molecular weight, composition and the presence of zinc.

The X-ray structure reveals that the molecule has the form of two globular entities, separated by a deep cleft. Within this cleft is the zinc atom, which is known to be necessary for enzymatic activity, but can be extracted by a chelating agent without destruction of the crystal. There are several internal helical segments. The zinc ligands are identified as two histidines and a glutamic acid; this is the same combination as in carboxypeptidase, which is in other structural respects quite different.

Other active site residues, which may be involved in catalysis, are a buried glutamate, an aspartate and an arginine associated in an ion pair, a tyrosine and a histidine bound by a salt link to another aspartate. The presence of calcium is a feature that thermolysin does not share with carboxypeptidase. There are four calcium binding sites, two of them joined in a cluster of five carboxylate groups which are partly buried. The other two sites are superficial, and also involve carboxyl groups. These calcium ions are not essential for activity, but are believed to confer thermal stability on the structure.

The structure in the presence of substrates or inhibitors has not yet been determined, so that no direct inferences about the enzymatic mechanism are at this stage possible. 